\title{
Does the magnetic field in the fluid core contribute a lot to Earth nutation?
}

\section{Cheng-Li Huang $^{1}$, Véronique Dehant ${ }^{2}$, Xin-Hao Liao ${ }^{1}$, Olivier de Viron ${ }^{2}$, and Tim van Hoolst ${ }^{2}$}

\author{
${ }^{1}$ Shanghai Astronomical Observatory, CAS, 80 Nandan Road, Shanghai 200030, P.R. China \\ email: clhuang,xhliao@shao.ac.cn \\ ${ }^{2}$ Royal Observatory of Belgium, Avenue Circulaire 3, B-1180 Brussels, Belgium \\ email: veronique.dehant,o.deviron,timvh@oma.be
}

Abstract. We discuss the influence of the magnetic field in the fluid core to the Earth's nutation.

Keywords. Earth, reference systems, magnetic fields

The existence of relative nutational motions between the liquid core and its surrounding solid parts induces a shearing of the magnetic field. An incremental magnetic field is then created, which perturbs the nutations themselves. This problem has already been addressed within a nutation model by Buffett (1992, Paper 1) and Buffett et al. (2002, Paper 2). These authors used an angular momentum budget approach, and their results are taken into account in the MHB nutation model.

In this work, the magnetic field influence is incorporated in the numerical integration method used in precise nutation theory. New equations for that problem and new boundary conditions inside the Earth are developed, and a new strategy to compute nutations is established. The Coriolis force, which is ignored in Paper 1 is included in our work. Our results show that the change of the free core nutation period is very consistent with the change in the main nutation (18.6 yr and retro-annual) terms.

Comparisons of these results with Paper 1 and 2 are made, and discussions on the contribution of the Coriolis force and the magnetic field itself on the coupling constants are also presented.

\section{References}

Buffett, B. A. 1992, J. Geophys. Res., 97, 19,581-19,597

Buffett, B. A., Mathews, P. M., \& Herring, T. 2002, J. Geophys. Res., 107, B4, 10.1029 / 2001JB000056 